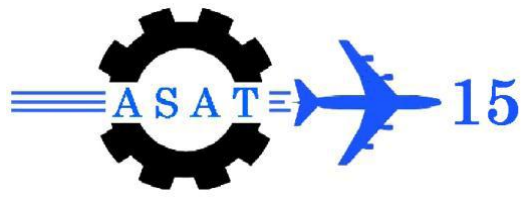

\title{
Concurrent Spacecraft Attitude and Orbit Estimation with Attitude Control Based on Magnetometer, Gyroscope, and GPS Measurements through Extended Kalman Filter
}

\author{
T. M. Habib*
}

\begin{abstract}
The main objective of this research is to provide attitude estimation, orbit estimation, and attitude control algorithms suitable for application to the next Egyptian scientific satellite. Concurrent spacecraft orbit and attitude estimates must be suitable for usage by the attitude control algorithm. The developed estimation algorithms are able to deal with sever tumbling conditions characterized by large initial attitude, angular velocity and position estimation errors. The estimation algorithms could provide attitude estimates within $0.5^{\circ}(3-\sigma)$ and $60 \mathrm{~m} \mathrm{(3- \sigma )}$ for the position estimation errors. The attitude control algorithm developed is able to bring the spacecraft from its initial tumbling conditions to nadir pointing within an error of only $0.5^{\circ}(3-\sigma)$.
\end{abstract}

Keywords: Attitude, orbit, control, estimation, EKF, GPS, Gyro, magnetometer.

\section{Introduction}

When the satellite leaves its launching vehicle it enters an operation mode called the detumbling mode. The detumbling mode is characterized by high angular velocities and large satellite attitude angles. The task of the attitude and orbit control system (AOCS) of an earth pointing satellite is to slow down this angular motion and bring the satellite to nadir pointing. To do so, the AOCS must implement suitable algorithms for attitude and orbit estimation with attitude control. These estimation algorithms should provide attitude and orbit estimates to the attitude control algorithm. Both estimation and control algorithms must be able to deal with large initial attitude angles and angular rates. Ref. [1] provided algorithms for spacecraft attitude estimation based on magnetometer measurements. But the results obtained were valid only for small attitude angles. Ref. [2] describes the process of magnetic attitude estimation of a tumbling spacecraft. The process didn't include solutions neither to the problems of the attitude control nor orbit estimation. Ref. [3] deals with the problem of attitude and orbit determination and control for a small geostationary satellite. Orbit estimation process isn't included in this study. In [4], the problem of spacecraft attitude and orbit estimation with attitude control is addressed but the estimation process was basically dominated by magnetometer measurements. The process of attitude estimation based on magnetometer measurements usually is characterized by slow convergence. Ref. [5], described the process of fast spacecraft orbit and attitude estimation, but it didn't include the process of attitude control. Ref. [6] discussed the process of spacecraft attitude estimation and control. But due to

Researcher, The Egyptian National Authority for Remote Sensing and Space Science, Cairo, Egypt; tamermekky@ hotmail.com 
the absence of orbit estimation process, the attitude angles converged slowly (typically after 3 orbits). Ref. [7] also didn't include the process of orbit estimation. In addition, the algorithms discussed were limited to coarse (not fine) attitude estimates (typically within $6^{\circ}$ ). Ref [8] discussed the problem of attitude estimation but the resulting attitude estimates hadn't been feedback to the control algorithm. Furthermore, the problem of orbit estimation isn't discussed at all.

The main objective of this research is to provide high accuracy attitude estimation, orbit estimation, and attitude control algorithms suitable for application to the next Egyptian scientific satellite during the detumbling and attitude acquisition modes. The estimation algorithms provided high accuracy estimates (typically within $0.5^{\circ} 3-\sigma$ for attitude estimates and $60 \mathrm{~m} \mathrm{3-} \sigma$ for the orbital estimates). To do so, the work done in [4], and [5], is extended to provide high accuracy fast converging attitude and orbit estimates needed by the attitude control algorithm. The provided algorithms are capable of dealing with high angular velocities and large attitude errors usually characterizing the detumbling and attitude acquisition modes. The attitude control algorithm presented is capable of bringing the satellite from the detumbling mode to the attitude acquisition mode within an error of only $0.5^{\circ}(3-\sigma)$. The measurement sensors utilized were, GPS receiver, magnetometer, and gyro. GPS, and magnetometer, measurements are used to provide estimates of the spacecraft orbital motion while as magnetometer and gyro measurements are used to provide estimates of spacecraft attitude.

\section{Modeling Spacecraft Attitude and Orbital Motion}

The first step to model the spacecraft orbital and attitude motion is to select the elements of the state vector. The state vector is selected to be

$$
X=\left[\begin{array}{lllllllllllll}
X_{I} & Y_{I} & Z_{I} & X_{I} & Y_{I} & Z_{I} & q_{1} & q_{2} & q_{3} & q_{4} & \omega_{x} & \omega_{y} & \omega_{z}
\end{array}\right]^{T}
$$

where

$\left[\begin{array}{lll}X_{I} & Y_{I} & Z_{I}\end{array}\right]$ : are the components of the sp acecraft position vector defined in the Earth Centered Inertial Coordinate System.

$\left[\begin{array}{lll}\stackrel{o}{X_{I}} & \stackrel{o}{ }_{I} & \stackrel{o}{Z_{I}}\end{array}\right]$ : are the components of the spacecraft velocity vector defined in the Earth Centered Inertial Coordinate System.

$\left[\begin{array}{llll}q_{1} & q_{2} & q_{3} & q_{4}\end{array}\right]$ : are the quaternion vector representing the rotation from Earth Centered Inertial Coordinate System to the Body Coordinate System.

$\left[\begin{array}{lll}\omega_{x} & \omega_{y} & \omega_{z}\end{array}\right]:$ are the components of the spacecraft Inertial angular velocity.

The orbital and attitude dynamics now could be written as [4] and [5]

$$
\stackrel{o}{X}=\left[\begin{array}{c}
\stackrel{o}{X_{o}} \\
o \\
X_{A}
\end{array}\right]=\left[\begin{array}{ll}
f_{11} & f_{12} \\
f_{21} & f_{22}
\end{array}\right]\left[\begin{array}{l}
X_{O} \\
X_{A}
\end{array}\right]+\left[\begin{array}{c}
B_{O} \\
B_{A}
\end{array}\right]+\eta=f(X)+B+\eta
$$




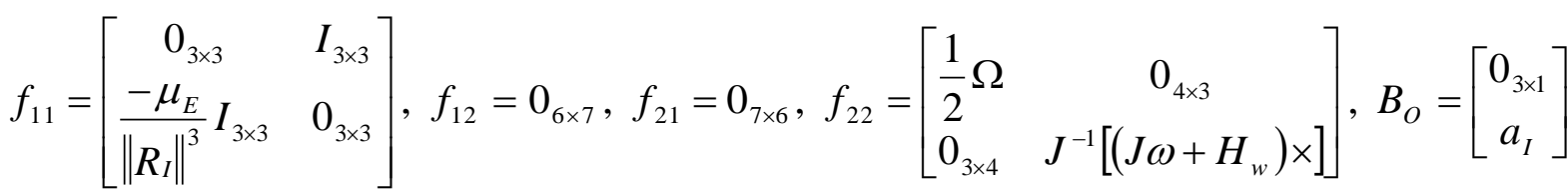

, and $B_{A}=\left[\begin{array}{c}0_{4 \times 1} \\ J^{-1} T\end{array}\right]$

where

$0_{i \times j} \quad:$ is an $i \times j$ zero matrix.

$I_{i \times j} \quad:$ is an $i \times j$ unit matrix.

$\mu_{E} \quad:$ is the earth's gravitational constant $\left(\mu_{E}=3.986 \times 10^{14} \mathrm{~m}^{3} / \mathrm{s}^{2}\right)$.

$\Omega \quad$ : is the skew symmetric matrix defined by

$\Omega=\left[\begin{array}{cccc}0 & \omega_{z} & -\omega_{y} & \omega_{x} \\ -\omega_{z} & 0 & \omega_{x} & \omega_{y} \\ \omega_{y} & -\omega_{x} & 0 & \omega_{z} \\ -\omega_{x} & -\omega_{y} & -\omega_{z} & 0\end{array}\right]$

$J \quad:$ is the spacecraft inertia tensor given as

$J=\left[\begin{array}{ccc}J_{x x} & -J_{x y} & -J_{x z} \\ -J_{y x} & J_{y y} & -J_{y z} \\ -J_{z x} & -J_{z y} & J_{z z}\end{array}\right]$.

$H_{w} \quad$ : is the angular momentum of the wheels.

$[\beta \times]:$ is the cross product matrix of $[\beta]=\left[\begin{array}{lll}\beta_{x} & \beta_{y} & \beta_{z}\end{array}\right]^{T}$ calculated from

$[\beta \times]=\left[\begin{array}{ccc}0 & -\beta_{z} & \beta_{y} \\ \beta_{z} & 0 & -\beta_{x} \\ -\beta_{y} & \beta_{x} & 0\end{array}\right]$

$a_{I} \quad:$ is the inertial acceleration.

$T \quad$ : is the Torque acting on the Spacecraft

$X_{O}=\left[\begin{array}{llllll}X_{I} & Y_{I} & Z_{I} & \stackrel{o}{X}_{I} & \stackrel{o}{Y}_{I} & \stackrel{o}{Z}_{I}\end{array}\right]^{T}$ and $X_{A}=\left[\begin{array}{llllllll}q_{1} & q_{2} & q_{3} & q_{4} & \omega_{x} & \omega_{y} & \omega_{z}\end{array}\right]^{T}$

$\eta \quad$ : is a zero mean Gaussian white-noise.

\section{Spacecraft Attitude Control}

There exists a quternion error vector which expresses the rotation from the spacecraft attitude direction in space, $q^{R \rightarrow B}$, and the target attitude direction toward which the satellite is oriented at the end of the attitude maneuver, $q_{T}$ [9]. The spacecraft attitude direction in space is parameterized by the attitude quternion representing the rotation from the reference coordinate system to the body coordinate system, $q^{R \rightarrow B}$. The reference coordinate system has its $\mathrm{x}$ axis pointing in the direction of the spacecraft velocity in its orbit, its $\mathrm{z}$ direction is nadir 
direction, and its y direction completes a right hand rule orthogonal coordinate system. The quaternion error vector is given by

$$
q_{E}=\left[\begin{array}{c}
q_{1 E} \\
q_{2 E} \\
q_{3 E} \\
q_{4 E}
\end{array}\right]=\left[\begin{array}{cccc}
q_{T 4} & q_{T 3} & -q_{T 2} & q_{T 1} \\
-q_{T 3} & q_{T 4} & q_{T 1} & q_{T 2} \\
q_{T 2} & -q_{T 1} & q_{T 4} & q_{T 3} \\
-q_{T 1} & -q_{T 2} & -q_{T 3} & q_{T 4}
\end{array}\right]\left[\begin{array}{c}
-q_{1}^{R \rightarrow B} \\
-q_{2}^{R \rightarrow B} \\
-q_{3}^{R \rightarrow B} \\
q_{4}^{R \rightarrow B}
\end{array}\right]
$$

The nonlinear control law is given by

$$
\begin{aligned}
& T_{c x r}=2 K_{x} q_{1 E} q_{4 E}+K_{x d} \omega_{B R x} \\
& T_{c y r}=2 K_{y} q_{2 E} q_{4 E}+K_{y d} \omega_{B R y} \\
& T_{c z r}=2 K_{z} q_{3 E} q_{4 E}+K_{z d} \omega_{B R z}
\end{aligned}
$$

where

$T_{c x r}, T_{c y r}$, and $T_{c z r}$ are control torques in the directions of the body axes triad system.

$K_{x}, K_{x d}, K_{y}, K_{y d}, K_{z}$, and $K_{z d}$ are the controller.

$\omega_{B R x}, \omega_{B R y}$, and $\omega_{B R z}$ are the angular velocities of the body frame with respect to the reference frame.

Note that the quaternion vector, $q^{R \rightarrow B}$, is to be provided by the estimation algorithm.

\section{Extended Kalman Filter}

The same structure of the extended Kalman filter found in [5] is utilized. The only difference exists in the measurement vector and its corresponding measurement matrix. The measurement vector is given by

$$
h=\left[\begin{array}{lllllllll}
b_{x b} & b_{y b} & b_{z b} & \omega_{x} & \omega_{y} & \omega_{z} & X_{I} & Y_{I} & Z_{I}
\end{array}\right]+v
$$

where $v$ is a zero mean Gaussian white noise. And the corresponding measurement matrix is given by

$$
\frac{\partial h}{\partial X}=\left[\begin{array}{ccccccccccccc}
\frac{\partial b_{x b}}{\partial X_{I}} & \frac{\partial b_{x b}}{\partial Y_{I}} & \frac{\partial b_{x b}}{\partial Z_{I}} & \frac{\partial b_{x b}}{\partial X_{I}} & \frac{\partial b_{x b}}{\partial Y_{I}} & \frac{\partial b_{x b}}{\partial Z_{I}} & \frac{\partial b_{x b}}{\partial q_{1}} & \frac{\partial b_{x b}}{\partial q_{2}} & \frac{\partial b_{x b}}{\partial q_{3}} & \frac{\partial b_{x b}}{\partial q_{4}} & \frac{\partial b_{x b}}{\partial \omega_{x}} & \frac{\partial b_{x b}}{\partial \omega_{y}} & \frac{\partial b_{x b}}{\partial \omega_{z}} \\
\frac{\partial b_{y b}}{\partial X_{I}} & \frac{\partial b_{y b}}{\partial Y_{I}} & \frac{\partial b_{y b}}{\partial Z_{I}} & \frac{\partial b_{y b}}{\partial X_{I}} & \frac{\partial b_{y b}}{o} & \frac{\partial b_{y b}}{o} & \frac{\partial b_{y b}}{\partial q_{1}} & \frac{\partial b_{y b}}{\partial q_{2}} & \frac{\partial b_{y b}}{\partial q_{3}} & \frac{\partial b_{y b}}{\partial q_{4}} & \frac{\partial b_{y b}}{\partial \omega_{x}} & \frac{\partial b_{y b}}{\partial \omega_{y}} & \frac{\partial b_{y b}}{\partial \omega_{z}} \\
\frac{\partial b_{z b}}{\partial X_{I}} & \frac{\partial b_{z b}}{\partial Y_{I}} & \frac{\partial b_{z b}}{\partial Z_{I}} & \frac{\partial b_{z b}}{\partial X_{I}} & \frac{\partial b_{z b}}{o} & \frac{\partial b_{z b}}{o} & \frac{\partial b_{z b}}{\partial q_{I}} & \frac{\partial b_{z b}}{\partial q_{2}} & \frac{\partial b_{z b}}{\partial q_{3}} & \frac{\partial b_{z b}}{\partial q_{4}} & \frac{\partial b_{z b}}{\partial \omega_{x}} & \frac{\partial b_{z b}}{\partial \omega_{y}} & \frac{\partial b_{z b}}{\partial \omega_{z}} \\
0 & 0 & 0 & 0 & 0 & 0 & 0 & 0 & 0 & 0 & 1 & 0 & 0 \\
0 & 0 & 0 & 0 & 0 & 0 & 0 & 0 & 0 & 0 & 0 & 1 & 0 \\
0 & 0 & 0 & 0 & 0 & 0 & 0 & 0 & 0 & 0 & 0 & 0 & 1 \\
1 & 0 & 0 & 0 & 0 & 0 & 0 & 0 & 0 & 0 & 0 & 0 & 0 \\
0 & 1 & 0 & 0 & 0 & 0 & 0 & 0 & 0 & 0 & 0 & 0 & 0 \\
0 & 0 & 1 & 0 & 0 & 0 & 0 & 0 & 0 & 0 & 0 & 0 & 0
\end{array}\right]
$$




$$
\begin{aligned}
& \frac{\partial b_{x b}}{\partial X_{I}}=A_{11} \frac{\partial b_{x I}}{\partial X_{I}}+A_{12} \frac{\partial b_{y I}}{\partial X_{I}}+A_{13} \frac{\partial b_{z I}}{\partial X_{I}} \\
& \frac{\partial b_{x b}}{\partial Y_{I}}=A_{11} \frac{\partial b_{x I}}{\partial Y_{I}}+A_{12} \frac{\partial b_{y I}}{\partial Y_{I}}+A_{13} \frac{\partial b_{z I}}{\partial Y_{I}} \\
& \frac{\partial b_{x b}}{\partial Z_{I}}=A_{11} \frac{\partial b_{x I}}{\partial Z_{I}}+A_{12} \frac{\partial b_{y I}}{\partial Z_{I}}+A_{13} \frac{\partial b_{z I}}{\partial Z_{I}} \\
& \frac{\partial b_{y b}}{\partial X_{I}}=A_{21} \frac{\partial b_{x I}}{\partial X_{I}}+A_{22} \frac{\partial b_{y I}}{\partial X_{I}}+A_{23} \frac{\partial b_{z I}}{\partial X_{I}} \\
& \frac{\partial b_{y b}}{\partial Y_{I}}=A_{21} \frac{\partial b_{x I}}{\partial Y_{I}}+A_{22} \frac{\partial b_{y I}}{\partial Y_{I}}+A_{23} \frac{\partial b_{z I}}{\partial Y_{I}} \\
& \frac{\partial b_{y b}}{\partial Z_{I}}=A_{21} \frac{\partial b_{x I}}{\partial Z_{I}}+A_{22} \frac{\partial b_{y I}}{\partial Z_{I}}+A_{23} \frac{\partial b_{z I}}{\partial Z_{I}} \\
& \frac{\partial b_{z b}}{\partial X_{I}}=A_{31} \frac{\partial b_{x I}}{\partial X_{I}}+A_{32} \frac{\partial b_{y I}}{\partial X_{I}}+A_{33} \frac{\partial b_{z I}}{\partial X_{I}} \\
& \frac{\partial b_{z b}}{\partial Y_{I}}=A_{31} \frac{\partial b_{x I}}{\partial Y_{I}}+A_{32} \frac{\partial b_{y I}}{\partial Y_{I}}+A_{33} \frac{\partial b_{z I}}{\partial Y_{I}} \\
& \frac{\partial b_{z b}}{\partial Z_{I}}=A_{31} \frac{\partial b_{x I}}{\partial Z_{I}}+A_{32} \frac{\partial b_{y I}}{\partial Z_{I}}+A_{33} \frac{\partial b_{z I}}{\partial Z_{I}}
\end{aligned}
$$

where $b_{. I}$ is the inertial component of the earth's magnetic field vector, and

$$
\left[\begin{array}{lll}
A_{11} & A_{12} & A_{13} \\
A_{21} & A_{22} & A_{21} \\
A_{31} & A_{32} & A_{33}
\end{array}\right]=\left[\begin{array}{ccc}
q_{1}^{2}-q_{2}^{2}-q_{3}^{2}+q_{4}^{2} & 2\left(q_{1} q_{2}+q_{3} q_{4}\right) & 2\left(q_{1} q_{3}-q_{2} q_{4}\right) \\
2\left(q_{1} q_{2}-q_{3} q_{4}\right) & -q_{1}^{2}+q_{2}^{2}-q_{3}^{2}+q_{4}^{2} & 2\left(q_{2} q_{3}+q_{1} q_{4}\right) \\
2\left(q_{1} q_{3}+q_{2} q_{4}\right) & 2\left(q_{2} q_{3}-q_{1} q_{4}\right) & -q_{1}^{2}-q_{2}^{2}+q_{3}^{2}+q_{4}^{2}
\end{array}\right]
$$

Also we have

$$
\begin{gathered}
\partial b_{x b} / \partial q_{1}=2 q_{1} b_{x I}+2 q_{2} b_{y I}+2 q_{3} b_{z I} \\
\partial b_{x b} / \partial q_{2}=-2 q_{2} b_{x I}+2 q_{1} b_{y I}-2 q_{4} b_{z I} \\
\partial b_{x b} / \partial q_{3}=-2 q_{3} b_{x I}+2 q_{4} b_{y I}+2 q_{1} b_{z I} \\
\partial b_{x b} / \partial q_{4}=2 q_{4} b_{x I}+2 q_{3} b_{y I}-2 q_{2} b_{z I} \\
\partial b_{Y b} / \partial q_{1}=2 q_{2} b_{x I}-2 q_{1} b_{y I}+2 q_{4} b_{z I} \\
\partial b_{Y b} / \partial q_{2}=2 q_{1} b_{x I}+2 q_{2} b_{y I}+2 q_{3} b_{z I} \\
\partial b_{Y b} / \partial q_{3}=-2 q_{4} b_{x I}-2 q_{3} b_{y I}+2 q_{2} b_{z I} \\
\partial b_{Y b} / \partial q_{4}=-2 q_{3} b_{x I}+2 q_{4} b_{y I}+2 q_{1} b_{z I} \\
\partial b_{Z b} / \partial q_{1}=2 q_{3} b_{x I}-2 q_{4} b_{y I}-2 q_{1} b_{z I} \\
\partial b_{Z b} / \partial q_{2}=2 q_{4} b_{x I}+2 q_{3} b_{y I}-2 q_{2} b_{z I} \\
\partial b_{Z b} / \partial q_{3}=2 q_{1} b_{x I}+2 q_{2} b_{y I}+2 q_{3} b_{z I} \\
\partial b_{Z b} / \partial q_{4}=2 q_{2} b_{x I}-2 q_{1} b_{y I}+2 q_{4} b_{z I}
\end{gathered}
$$




\section{Observability and Controllability Analysis}

Check of the observability and controllability matrices could be done through the computation of the observability and controllability matrices. The observability matrix, $O B$, is given by

$$
O B=\left[\begin{array}{c}
H_{k} \\
H_{k} A_{k} \\
H_{k} A_{k}^{2} \\
\vdots \\
H_{k} A_{k}^{12}
\end{array}\right]
$$

And the controllability matrix is given by

$$
C O=\left[\begin{array}{lllll}
G & A_{k} G & A_{k}^{2} G & \cdots & A_{k}^{12} G
\end{array}\right]
$$

where the matrix, $G$, is given by

$$
G=\left[\begin{array}{cc}
0_{3 \times 3} & 0_{3 \times 3} \\
I_{3 \times 3} & 0_{3 \times 3} \\
0_{4 \times 3} & 0_{4 \times 3} \\
0_{3 \times 3} & J^{-1}
\end{array}\right]
$$

Thus, for complete controllability and observability the controllability and observability matrices must have a full rank.

\section{A Simulation Case Study}

In order to verify the developed methodologies, a case study spacecraft is utilized. The spacecraft initial conditions are $: a$ (semi major axis) $=7189200 \mathrm{~m}, e$ (orbit eccentricity) = $0.01, i$ (orbit inclination $)=100.585^{\circ}, \Omega$ (right ascension of ascending node $)=339.5^{\circ}, \vartheta$ (argument of perigee) $=69^{\circ}$, and $v$ (true anomaly) $=3.5^{\circ} . \phi$ (roll angle $)=170^{\circ}, \psi($ yaw angle) $=-165^{\circ}$, and $\theta$ (pitch angle) $=60^{\circ}$. The inertial angular velocity components are $\omega_{x}=$ $4 \% \mathrm{sec}, \omega_{y}=4 \% \mathrm{sec}$, and $\omega_{z}=-2 \%$ sec. The gyroscope utilized is assumed to be Inertial Reference Unit REGYS 30/ REGYS 3S produced by Sagem. The gyroscope error model is assumed to be given by equation (7) (i.e., white Gaussian noise). The problem of gyroscope bias estimation is assumed to be solved by a separate estimation process. The GPS receiver position measurement accuracy is $50 \mathrm{~m}(3-\sigma)$. Spacecraft moments of inertia are $\mathrm{I}_{\mathrm{x}}=11.2$, $\mathrm{I}_{\mathrm{y}}=11.4, \mathrm{I}_{\mathrm{z}}=9.2, \mathrm{I}_{\mathrm{xy}}=0.02, \mathrm{I}_{\mathrm{xz}}=-0.08, \mathrm{I}_{\mathrm{yz}}=0.2 \mathrm{~kg} \cdot \mathrm{m}^{2}$.

For the roll, pitch, yaw, and body angular velocities, their values are not known so they could be assumed logically equal to zero. For the remaining values, numbers are assumed in order to test the estimator ability to converge. Thus, the estimator initial conditions are : $a$ (semi major axis $)=7039200 \mathrm{~m}, e$ (orbit eccentricity $)=0, i$ (orbit inclination $)=98.085^{\circ}, \Omega$ (right ascension of ascending node $)=337.5^{\circ}, \vartheta($ argument of perigee $)=69^{\circ}$, and $v$ (true anomaly) $=0^{\circ} . \phi($ roll angle $)=0^{\circ}, \psi($ yaw angle $)=0^{\circ}$, and $\theta($ pitch angle $)=0^{\circ}$. The inertial angular velocity components are $\omega_{x}=0 \% \mathrm{sec}, \omega_{y}=-0.0011 \% \mathrm{sec}$, and $\omega_{z}=0 \% \mathrm{sec}$. Fig. 1 . represents the time history of the spacecraft attitude angles. As seen in this figure, the initial attitude angles were as large 170 degree. The control algorithm discussed is capable of dealing with these large angles which are encountered during the detumbling mode. Fig.2. shows the time history of the spacecraft inertial angular velocities. As clear in Fig.1 and Fig.2, the attitude control algorithm succeeded in bringing the satellite from the detumbling mode to nadir pointing in less than half of an orbit. Fig. 3. displays the time history of the spacecraft attitude 
estimation error of the pitch, roll, and yaw angles respectively. The maximum estimation error was about $0.5^{\circ}(3-\sigma)$. Fig. 4 represents variation of the earth's magnetic field measurement error. The initial magnitude of the position estimation error was about $452 \mathrm{Km}$. After only one time step this error has been reduced drastically to $45 \mathrm{~m}(3-\sigma)$. Fig. 5 shows the time history of the observability matrix rank. As shown in this figure, the rank of the observability matrix is always equal to 13 , which indicates full observability of the system states. Fig. 6 represents the time history of the controllability matrix rank. As clear in this figure, the system is fully controllable due to the full rank of the controllability matrix.
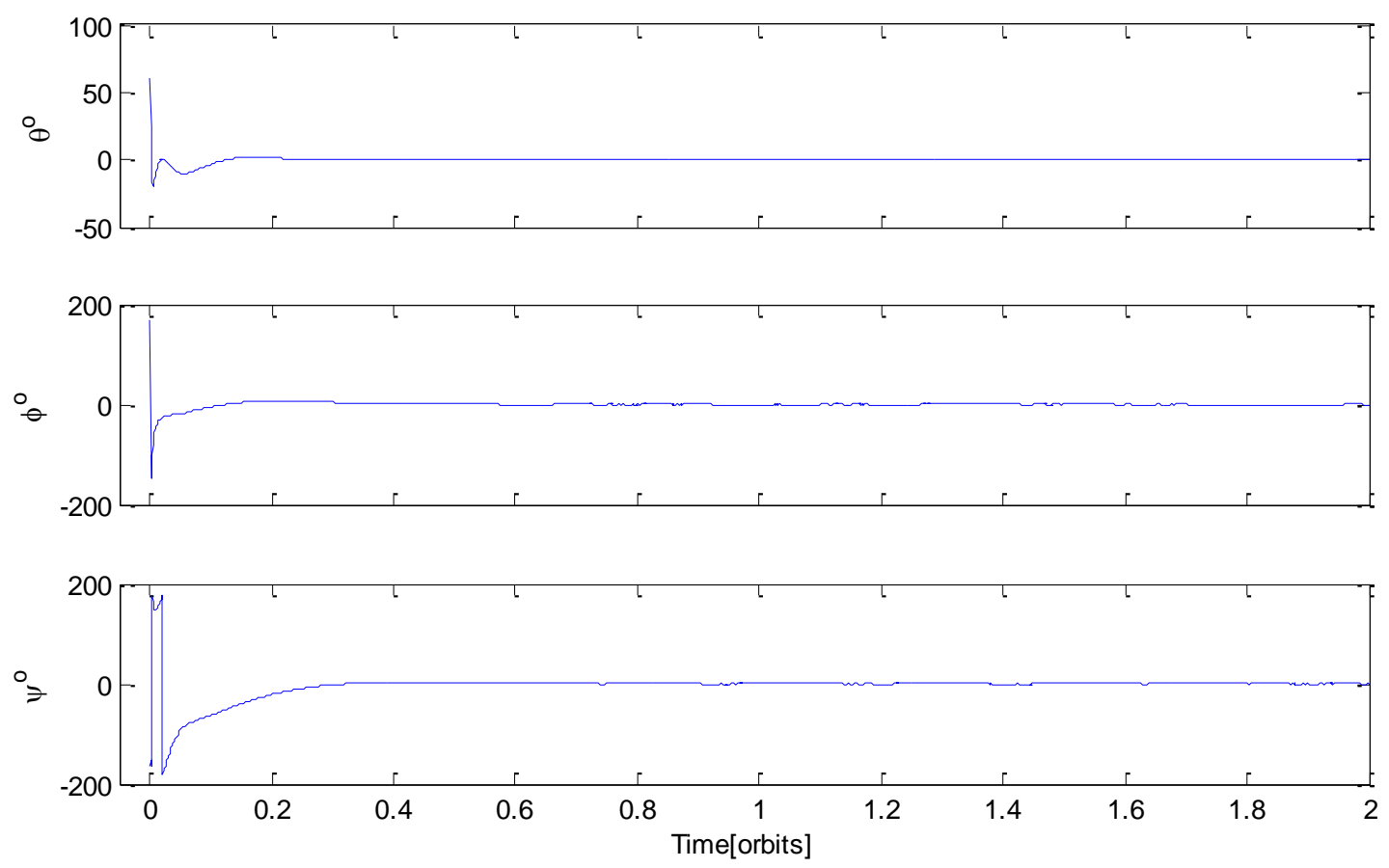

Fig. 1. Spacecraft attitude time history
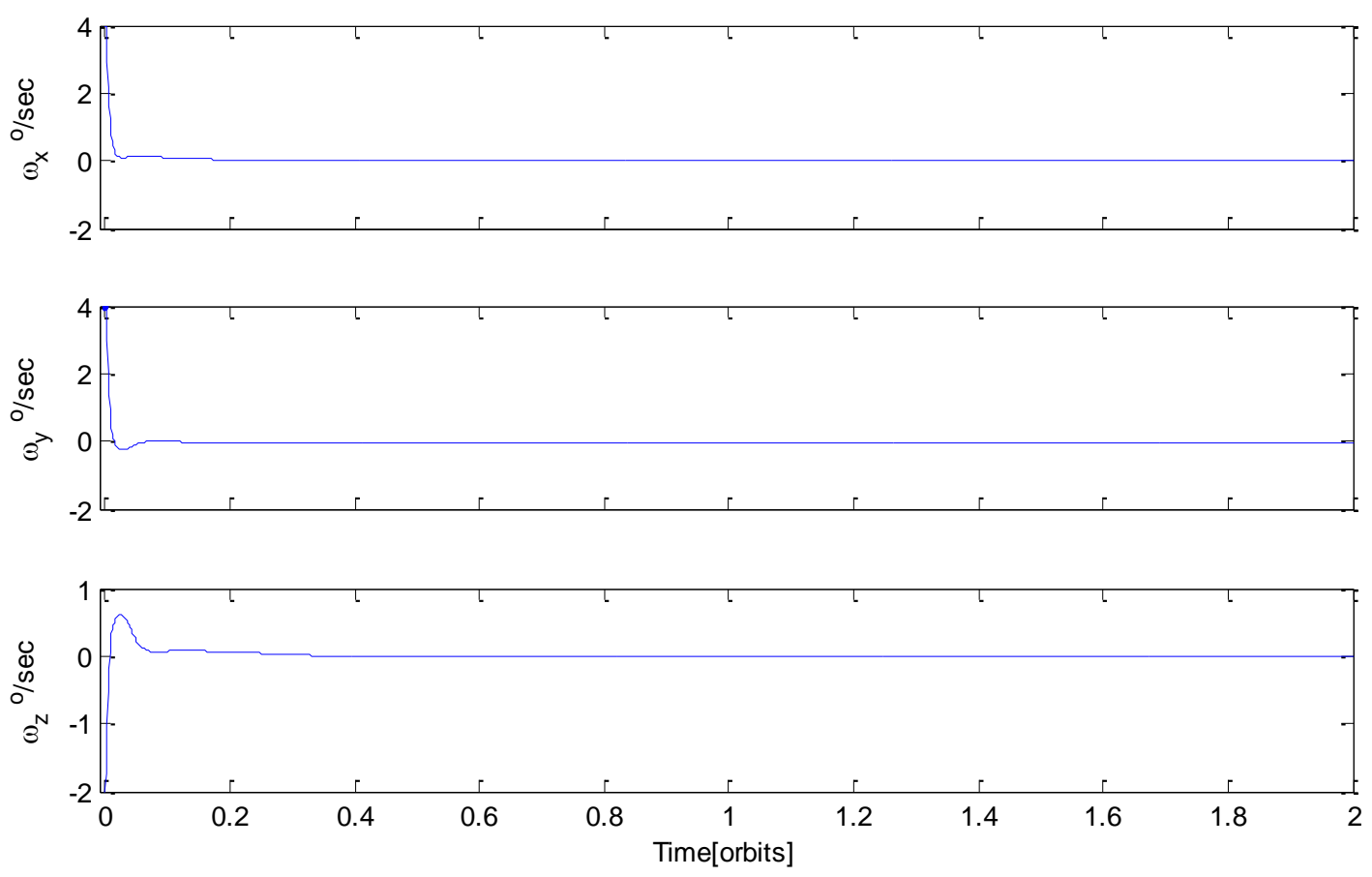

Fig. 2. Spacecraft inertial angular velocity time history. 

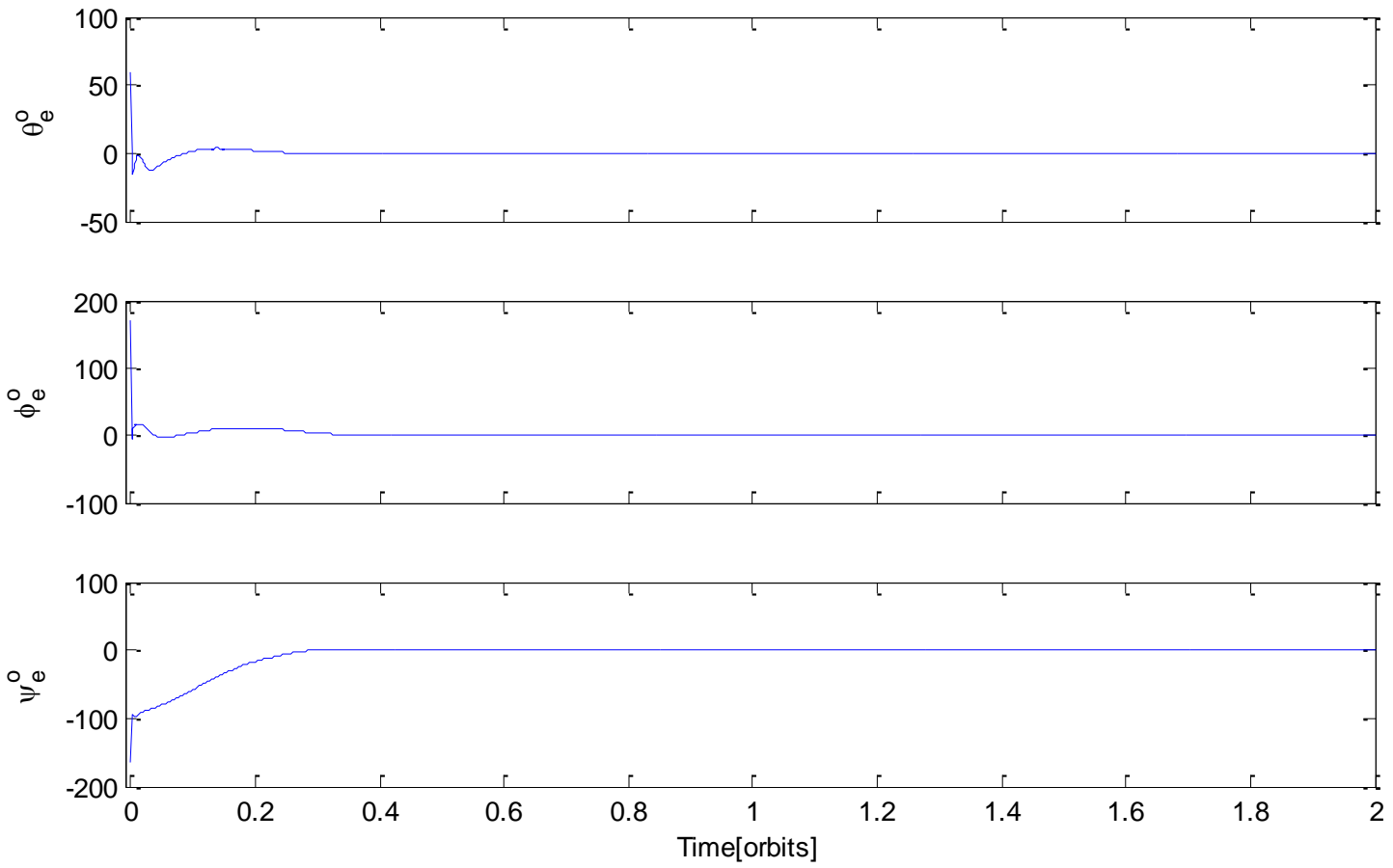

Fig. 3. Spacecraft attitude estimation error time history.

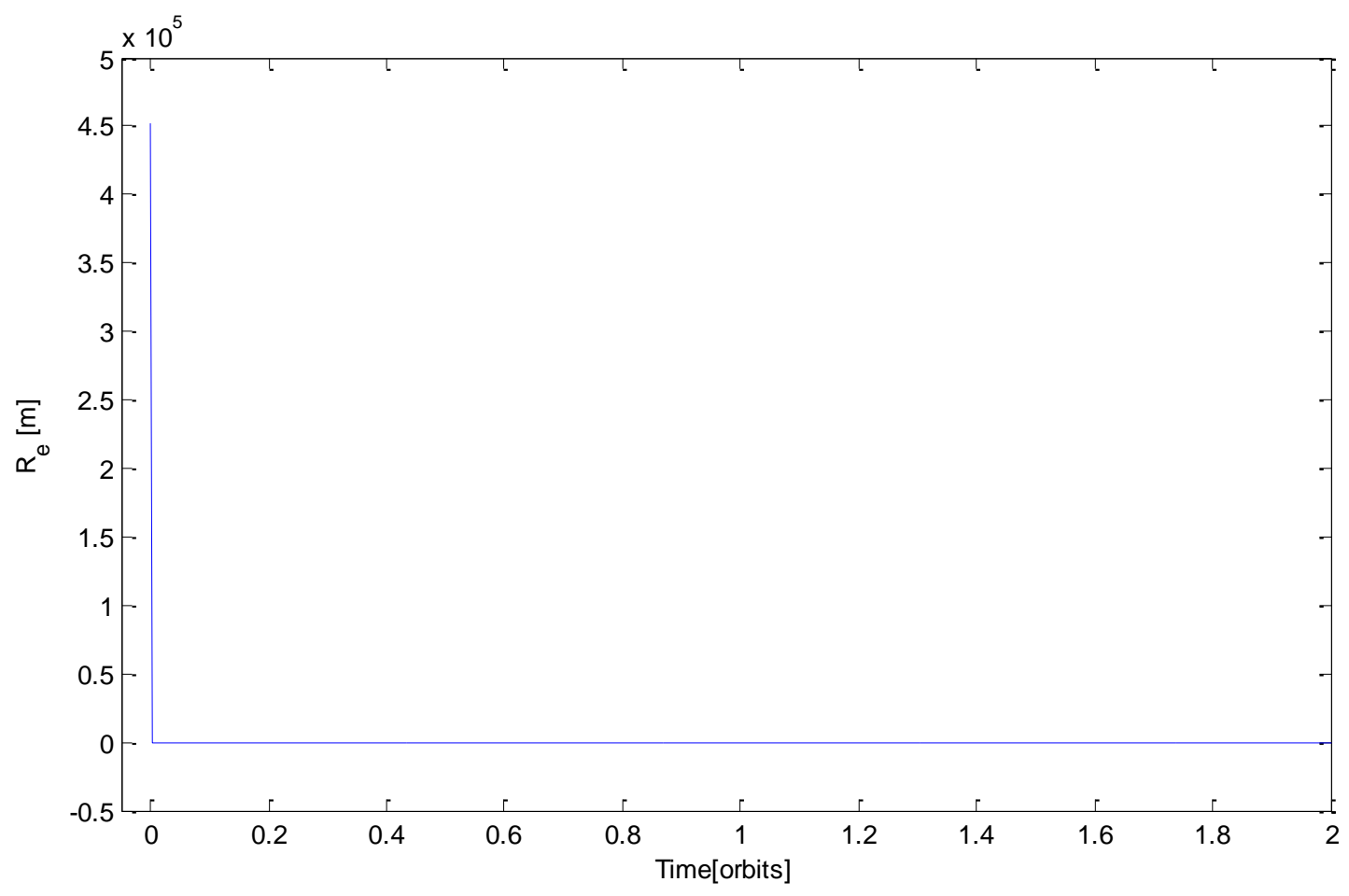

Fig. 4. Position estimation error time history. 


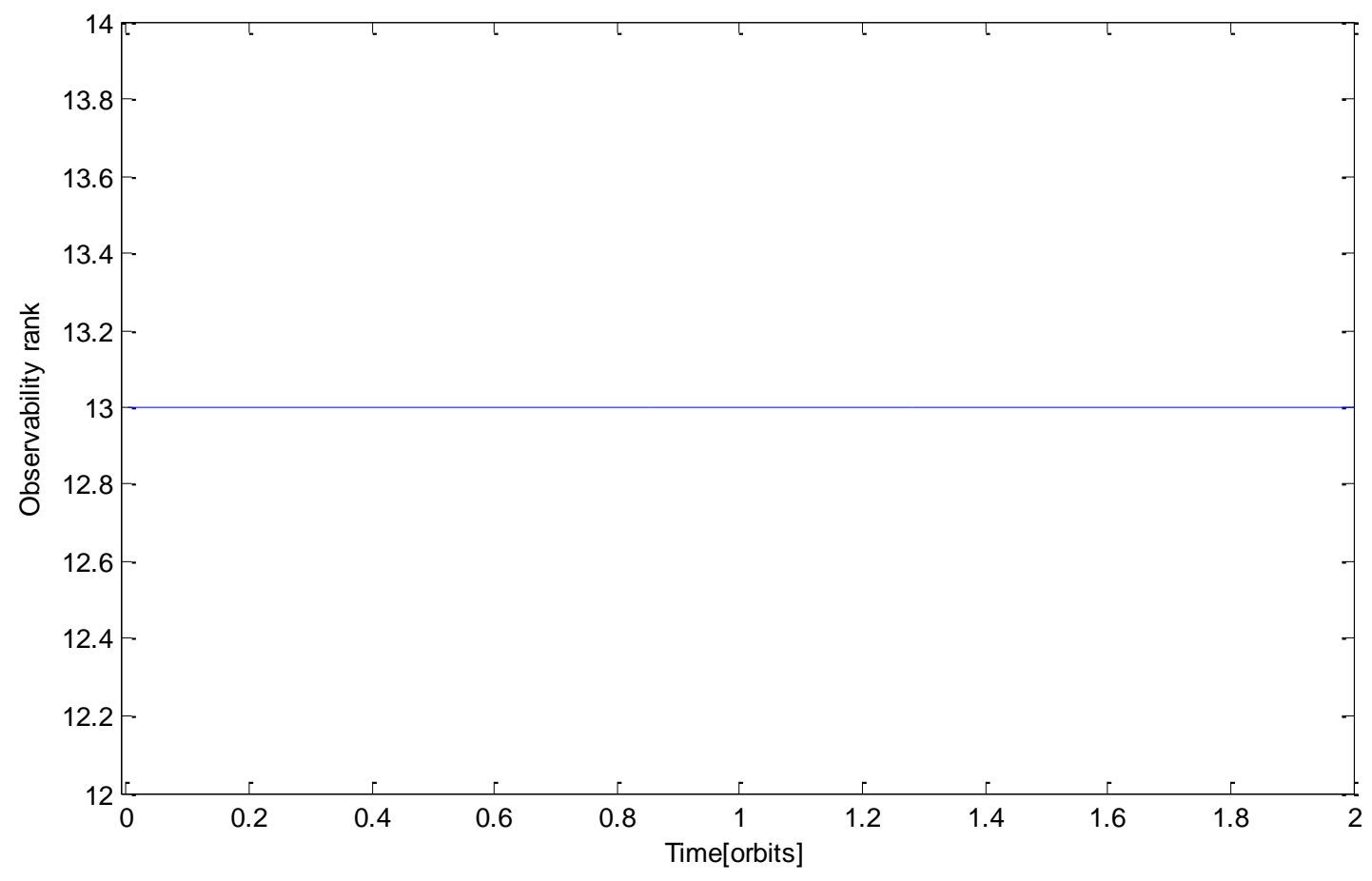

Fig. 5 Time history of the observability matrix rank

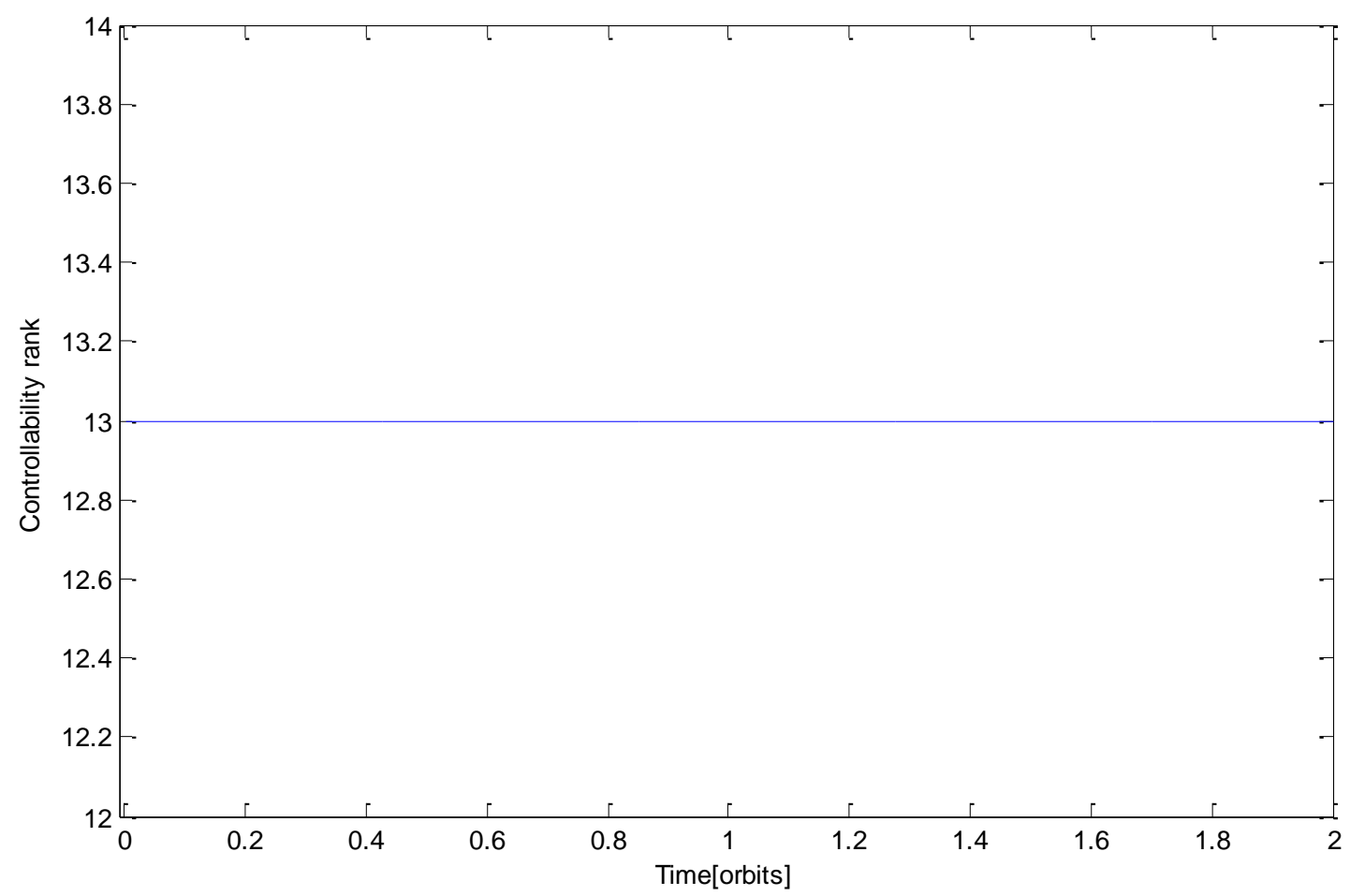

Fig.6. Time history of the controllability matrix rank.

Table (1) shows a comparison between the current research results and the results of reference [5] 
Table.1 A comparison between the algorithms developed in reference [5], and current research algorithm.

\begin{tabular}{l|l|l}
\hline \hline & Algorithm developed in [5] & Current research algorithm \\
\hline Sensors utilized & $\begin{array}{l}\text { Star sensor, magnetometer, } \\
\text { gyroscope, and GPS }\end{array}$ & $\begin{array}{l}\text { Magnetometer, gyroscope, } \\
\text { and GPS }\end{array}$ \\
\hline Satellite operation mode & High accuracy operation mode & $\begin{array}{l}\text { Detumbling, stand-by mode, } \\
\text { and high accuracy operation } \\
\text { modes }\end{array}$ \\
\hline $\begin{array}{l}\text { Attitude and orbit estimates } \\
\text { are fed back to the attitude } \\
\text { control algorithm }\end{array}$ & $\begin{array}{l}\text { No, because there is no } \\
\text { attitude control algorithm. }\end{array}$ & Yes \\
\hline \hline
\end{tabular}

\section{Conclusion}

The methods of spacecraft orbit and attitude estimation during the detumbling and attitude acquisition modes had worked effectively with each other despite of large initial attitude and orbit estimation errors. The estimation error was about $0.5^{\circ}(3-\sigma)$ for the attitude angles and $60 \mathrm{~m}(3-\sigma)$ for the position estimation error. Both estimates of spacecraft attitude and orbit are fed successfully to the attitude control algorithm. The attitude control algorithm was able to bring the satellite from the detumbling mode to nadir pointing during less than half of an orbit within accuracy of $0.5^{\circ}(3-\sigma)$. The rank of the observability and controllability matrices was thirteen, which is indicating a full rank, so the plant is considered to be fully observable and controllable.

\section{References}

[1] Bak, T., "Spacecraft Attitude Determination- a Magnetometer Approach", PhD Thesis, Aalborg University, 1999.

[2] Sturm, E., "Magnetic Attitude Estimation of a Tumbling Spacecraft", MSc Thesis, California Polytechnic State University, 2005.

[3] Thopil, G., "An Attitude and Orbit Determination and Control System for a Small Geostationary Satellite", MSc Thesis, Stellenbosch, 2006.

[4] Tamer, M., "New Algorithms of Nonlinear Spacecraft Attitude Control via Attitude, Angular velocity, and Orbit Estimation Based on the Earth's Magnetic Field", PhD Thesis, Cairo University, 2009.

[5] Tamer, M., "Fast Converging with High Accuracy Estimates of Satellite Attitude and Orbit Based on Magnetometer Augmented with Gyro, Star Sensor and GPS via Extended Kalman Filter", The Egyptian Journal of Remote Sensing and Space Sciences, , Vol.14, Issue 2, pp. 57-61, 2011.

[6] Mohammad, A., and Sang-Young, P., "Integrated attitude determination and control system via magnetic measurements and actuation", Acta Astronautica, Vol.69, pp. 168$185,2011$.

[7] Tian, X., et al., "Design and on-orbit performance of the attitude determination and control system for th eZDPS-1A pico-satellite", Acta Astronautica, Vol.77, pp. 182-196, 2012 .

[8] Tang, X., et al., "Square-root quaternion cubature Kalman filtering for spacecraft attitude estimation", Acta Astronautica, Vol.76, pp. 84-94, 2012.

[9] Sidi, M. J., "Spacecraft Dynamics and Control, a Practical Engineering Approach", Cambridge University Press, 1997. 